\title{
Revision of EBG Metamaterials and Active Antennas
}

\author{
Gonzalo Expósito-Domínguez*, José Manuel Fernández-González*, Pablo Padilla†, \\ Manuel Sierra-Castañer* \\ * Radiation Group, Signals, Systems and Radiocommunications, Universidad Politécnica de Madrid, 28040, Spain \\ Email: \{gexposito, jmfdez, mscastaner\}@gr.ssr.upm.es \\ $\dagger$ Department of Signal Theory, Telematics and Communications, Universidad de Granada, Granada 18071, Spain. \\ Email: pablopadilla@ugr.es
}

\begin{abstract}
This review of Electromagnetic Band Gap (EGB) metamaterials and steering integrated antennas was carried out in IMST GmbH under a Short Term Scientific Mission. This activity is in line with Coordinating the Antenna Research in Europe (CARE). The aim is to identify the newest trends, and suggest novel solutions and design methodologies for various applications.
\end{abstract}

\section{INTRODUCTION}

Starting from the results achieved by the ACE Network of Excellence (from 2004 to 2008), CARE (introduced in 2009), continues and reinforces the collaboration among the European Institutions involved in antenna research. In CARE, the level of cooperation reached in ACE will be sustained and improved by students and researchers secondment, best practices sharing, industrial training and dissemination, by publications and conferences.

This paper gives an overview of the EBG metamaterials theory in section II [1]. The section III is devoted to EBG applications. In section IV the main problems of steering integrated antennas are presented. Finally, in section $\mathrm{V}$ the conclusions are drawn.

\section{EBG THEORY}

Electromagnetic Band Gap (EBG) based on Frequency Selective Surfaces (FSS) [2] are one type of metamaterials with electrical properties [3].

EBG technique appears as an application of truncated frequency selective surface (FSS) [4]. These structures consist of an array of metal protrusions on a flat metal sheet and can be visualized as mushrooms protruding from the surface. When the period is small compared to the wavelength of interest, it is possible to analyze the material as an effective medium, with a surface impedance. These "mushrooms" present very high impedance for vertical an horizontal modes at certain frequencies.

These structures can be analyzed as resonant LC circuits, in which the capacitance is provided by the proximity of the metal plates:

$$
C=\left[\frac{w \varepsilon_{0}\left(\varepsilon_{e f f}\right)}{\pi}\right] \cosh ^{-1}\left(\frac{2 w}{g_{0}}\right)
$$

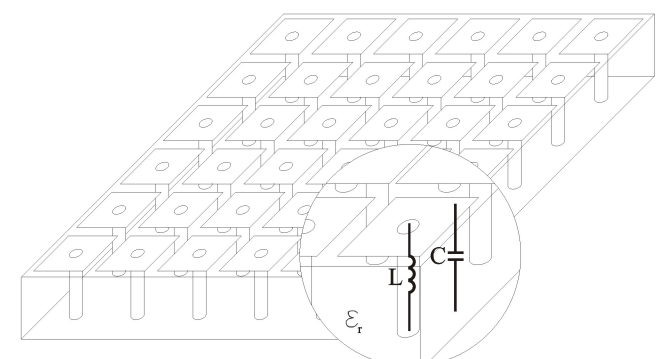

Fig. 1. High impedance surface and its model with parallel resonant LC circuit.

and the inductance is related to the thickness of the structure:

$$
L=\mu_{0} t
$$

Therefore, the surface impedance is given by the following expression:

$$
Z_{s}=\frac{j \omega L}{1-\omega^{2} L C}
$$

The resonance frequency of the circuit is given by:

$$
\omega_{0}=\frac{1}{\sqrt{L C}}
$$

Below resonance, the surface is inductive meanwhile above resonance, the surface is capacitive. Near $\omega_{0}$, the surface impedance is much higher than the impedance of free space [4].

\section{EBG APPLICATIONS}

Among others, EBG structures are used as ground planes emulating Artificial Magnetic Conductors (AMC) in a narrow frequency range. EBG are used also to reduce the mutual coupling between elements.

\section{A. EBG Ground plane}

EBG structure has one important feature: the in-phase reflection coefficient for plane waves. This property can be used to design low-profile wire antennas [5]. The low-profile 
TABLE I

COMPARISON OF PEC, PMC, AND EBG GROUND PLANES FOR LOW PROFILE ANTENNA DESIGNS.

\begin{tabular}{|l|l|l|}
\hline Ground plane & Reflection phase & Comments \\
\hline \hline PEC & $180^{\circ}$ & Reverse image. \\
\hline PMC & $0^{\circ}$ & Mutual coupling. \\
\hline EBG & $\begin{array}{l}\text { Varies from } 180^{\circ} \text { to }-180^{\circ} \\
\text { with frequency }\end{array}$ & $\begin{array}{l}\text { Suitable } \\
\text { frequency band. }\end{array}$ \\
\hline
\end{tabular}

design usually refers to the antenna structures whose height is less than one-tenth. In chapter 12 of [6], a comparison between PEC, PMC, and EBG ground planes is carried out. The conclusions are drawn in Table I.

In [7] EBG ground plane is used to reduce the effect of multipath by blocking the propagation of surface waves and cross polarization component in a low profile GALILEO antenna. Its performance is similar to a classical choke ring antenna, but with the advantage of low weight and low profile.

\section{B. Filters}

Thanks to its frequency selective feature, this EBG structure can be used as a filter. By applying three elements as a ground plane for a microstrip line, in [8] isolation higher than $55 \mathrm{~dB}$ in the first frequency band $(2.1 \mathrm{GHz})$ and $40 \mathrm{~dB}$ in the second one $(2.45 \mathrm{GHz})$ are achieved.

\section{Mutual coupling reduction}

Patch antennas are found to have very strong mutual coupling due to the pronounced surface waves on a thick and high permittivity substrates. However, in order to reduce antenna size and bandwidth enhancement there is no other option. In [9], four columns of EBG patches are inserted between the antennas in a $\epsilon_{r}=10.2$ and thickness $(h=2$ $\mathrm{mm})$ substrate. Finally $8 \mathrm{~dB}$ of reduction are obtained. In array applications where the separation between elements is $0.5 \lambda$ in order to avoid grating lobes, the available space is not enough to introduce four columns of EBG structures. In [10] different substrates are combined, radiating elements are suspended over a thick foam layer in order to increase the bandwith, meanwhile EBG structures are printed in a thin high permittivity substrate for size reduction and surface wave suppression. In [11] by using edge-located vias the size of mushroom-type EBG is reduced by $20 \%$. Among other strategies in [12] a fork shape is used. The area occupied by the fork-like structure is less than $40 \%$ of the mushroom-like structure. Besides, Microelectromechanical Systems (MEMS) are used and reconfigurable stop band is obtained.

Another technique studied is metal strips. Basically, the idea is to combine the EBG concept with soft surfaces. A comparison of bandgaps of mushroom-type EBG surface and corrugated and strip-type soft surfaces is shown in [13]. This stripe-type is used in [14] and in [15] to reduce mutual coupling. Finally, dual band planar soft surfaces are developed in [16], two sizes of strips are mixed in order to get dual forbidden band.

New solutions for patch size reduction are proposed. In order to maintain the working frequency with the same substrate thickness, the only parameter available is the capacitance $C$. In order to increase this parameter a multilayered structure is presented in Fig. 2(a) and Fig. 2(b) [17].

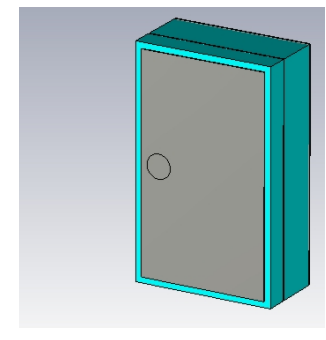

(a) Multilayered patch-shape

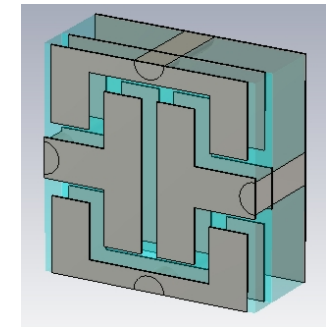

(b) Multilayered H-shape
Fig. 2. Multilayered mushroom-type solutions.

\section{STEERING APPLICATIONS}

The antenna array concept arises due to the necessity of getting higher directivity. By changing the phase feeding of the array elements the radiation pattern can be steered in the desired direction [18] without moving the antenna physically.

\section{A. Active networks}

This kind of feeding can be done through active networks such as phase shifters or microelectromechanical systems (MEMS). Those systems have high complexity but high flexibility and features. In [19] MEMS are used in an antenna array front end at $24 \mathrm{GHz}$. The aim of the project is to combine MEMS with monolithic microwave integrated circuits (MMIC), this system presents low insertion loss, large bandwidth, and good linearity over frequency. In [20] four elements array is fed by coplanar transmission lines (TL). Using MEMS, those TLs change their capacity and propagation velocity, therefore the radiating elements can be fed with the desired phase shift. In [21], a complex system of 156 radiating elements in $\mathrm{Ku}$ band is presented. This system is constructed in Low Temperature Co-Fired Ceramic (LTCC) technology, it has linear polarization with tracking, a scan range from $20^{\circ}$ to $60^{\circ}$ in elevation from horizon and $0^{\circ}$ to $360^{\circ}$ in azimuth. The antenna size is $20 \mathrm{~cm} \times 20 \mathrm{~cm} \times 2 \mathrm{~cm}$, optimum for car integration. In [22]-[23] the Smart Antenna Terminal (SANTANA) is presented. The system works in Kaband for reception (RX) and transmission (TX). The uplink frequency is $29.75 \mathrm{GHz}$ and the downlink frequency is 19.95 $\mathrm{GHz}$, this system has the capability of electronic steering in azimuth and elevation and the radiating element is realized in a 16 multilayered LTCC structure. L-band phased array for maritime satcom is shown in [24]. This antenna consists of 26 patch elements that are mounted on a soccer-like spherical aperture. This design is an innovative and powerful alternative to current mechanically steered systems. The last example, antenna GEODA-GRUA [25] is one conformal adaptative 
antenna designed for satellite communications. Operating at 1.7 $\mathrm{GHz}$ with circular polarization, it is possible to track and communicate with several satellites at once being able to receive signals in full azimuth and within the range of $5^{\mathrm{o}}$ to broadside elevation. The antenna is composed of 2700 radiating elements based on a set of 60 triangular arrays that are divided in 15 subarrays of 3 radiating elements.

\section{B. Passive networks}

Another beam control option is passive networks. Butler matrix network [26] consists in $2^{n}$ inputs, $2^{n}$ outputs, $2^{n-1} \log _{2} 2^{n}$ hybrid couplers, crossovers and phase shifters. The function of a Butler matrix is to combine signals in phase going or coming from an antenna array. It produces $2^{n}$ beams with constant angular separation. Blass matrix network [27] has two groups of transmission lines which are interconnected through hybrid couplers. The path difference, is the clue in order to control the beam steering. This solutions do not have so good features but are cheaper solutions. In [28] a dual circular polarized steering antenna for satellite communications in $\mathrm{X}$ band is presented. This antenna has the following capabilities: broadband capacity $(7.25-8.15 \mathrm{GHz}) 15 \%$, dual circular polarization (RHCP and LHCP, interchangeable for TX and RX), good axial ratio $(\mathrm{AR}<3 \mathrm{~dB})$. Finally this antenna is able to steer in elevation to $45^{\circ}, 75^{\circ}, 105^{\circ}$ and $135^{\circ}$ electronically with a Butler matrix network and $360^{\circ}$ in azimuth with a motorized junction. In [29], a system based in horn antennas and beamforming networks is shown. This antenna is capable of using several beams simultaneously. On the other hand, solutions based in Traveling Wave Antennas (TWA) [30]-[31], allow to control the direction of the radiation pattern as a function of frequency for a narrow band.

\section{CONCLUSIONS}

During this Short Term Scientific Mission, in IMST under the supervision and guidance of Dr. Marta Martínez-Vázquez a review of metamaterials and active antennas was carried out. The focus of the study was EBG structures and its applications. In future works EBG structures will be applied to reduce mutual coupling between elements in steering applications.

\section{ACKNOWLEDGMENT}

This work has been carried out in IMST GmbH in a Short Term Scientific Mission of COST ic0603, this stay was cosupported by CARE. This thesis is been supported by an UPM grant $\mathrm{CH} / 003 / 2011$, the CROCANTE project with reference TEC2008-06736-C03-01, and the SICOMORO project with reference TEC2011-28789-C02-01.

\section{REFERENCES}

[1] P.-S. Kildal. Definition of artificially soft and hard surfaces for electromagnetic waves. Electronics Letters, 24(3):168 -170, feb 1988.

[2] D. Sievenpiper, L. Zhang, R. Broas, N. Alexopoulos, and E. Yablonovitch. High-impedance electromagnetic surfaces with a forbidden frequency band. IEEE Trans. On Microwave Theory and Techniques, 47:2059-2074, Nov. 1999.

[3] T. Itoh C. Caloz. Electromagnetic metamaterials: transmission line theory and microwave applications: the engineering approach. John Wiley and Sons, 2006.
[4] F. Yang and Y. Rahmat-Samii. Electromagnetic Band Gap Structures in Antenna Engineering. The Cambridge RF and Microwave Engineering Series, 2008.

[5] Fan Yang and Y. Rahmat-Samii. Reflection phase characterizations of the ebg ground plane for low profile wire antenna applications. Antennas and Propagation, IEEE Transactions on, 51(10):2691 - 2703, oct. 2003.

[6] N. Engheta and R. Ziolkowski. Metamaterials, physics and engineering explorations. John Wiley and Sons, 2006.

[7] R. Baggen, M. Martinez-Vazquez, J. Leiss, S. Holzwarth, L.S. Drioli, and P. de Maagt. Low profile galileo antenna using ebg technology. Antennas and Propagation, IEEE Transactions on, 56(3):667 -674 march 2008.

[8] L. Inclan-Sanchez, J.-L. Vazquez-Roy, and E. Rajo-Iglesias. High isolation proximity coupled multilayer patch antenna for dual-frequency operation. Antennas and Propagation, IEEE Transactions on, 56(4):1180 -1183 , april 2008.

[9] Fan Yang and Y. Rahmat-Samii. Microstrip antennas integrated with electromagnetic band-gap (ebg) structures: a low mutual coupling design for array applications. Antennas and Propagation, IEEE Transactions on, 51(10):2936-2946, Oct. 2003.

[10] E. Rajo-Iglesias, O. Quevedo-Teruel, and L. Inclán-Sánchez. Mutual coupling reduction in patch antenna arrays by using a planar ebg structure and a multilayer dielectric substrate. IEEE Trans. on Antennas and Propagation, 56(6):1648-1655, Jun. 2008.

[11] E. Rajo-Iglesias, L. Inclan-Sanchez, J.-L. Vazquez-Roy, and E. GarciaMuoz. Size reduction of mushroom-type ebg surfaces by using edgelocated vias. Microwave and Wireless Components Letters, IEEE, 17(9):670 -672, sept. 2007.

[12] Li Yang, Mingyan Fan, Fanglu Chen, Jingzhao She, and Zhenghe Feng. A novel compact electromagnetic-bandgap (ebg) structure and its applications for microwave circuits. Microwave Theory and Techniques, IEEE Transactions on, 53(1):183 - 190, jan. 2005.

[13] E. Rajo-Iglesias, M. Caiazzo, L. Inclan-Sanchez, and P.-S. Kildal. Comparison of bandgaps of mushroom-type ebg surface and corrugated and strip-type soft surfaces. Microwaves, Antennas Propagation, IET, 1(1):184-189, february 2007.

[14] E. Rajo-Iglesias, O. Quevedo-Teruel, and L. Inclan-Sanchez. Planar soft surfaces and their application to mutual coupling reduction. Antennas and Propagation, IEEE Transactions on, 57(12):3852 -3859, dec. 2009.

[15] O. Quevedo-Teruel, L. Inclan-Sanchez, and E. Rajo-Iglesias. Soft surfaces for reducing mutual coupling between loaded pifa antennas. Antennas and Wireless Propagation Letters, IEEE, 9:91 -94, 2010.

[16] E. Rajo-Iglesias, J.-L. Vazquez-Roy, O. Quevedo-Teruel, and L. InclanSanchez. Dual band planar soft surfaces. Microwaves, Antennas Propagation, IET, 3(5):742 -748, august 2009.

[17] G. Expósito-Domínguez, P. Padilla-Torre, J. M. Fernández-González, and M. Sierra-Castañer. Mutual coupling reduction techniques in electronic steering antennas in $\mathrm{x}$ band. $33^{\text {rd }}$ ESA antenna workshop on challenges for space antenna systems, Oct. 2011.

[18] C. A. Balanis. Antenna Theory, analysis and design. Wiley india, third edition edition, 1997.

[19] M.A. Campo, O. Litschke, T. Vaha-Heikkila, L. Markku, and R. Baggen. Mems-4-mmic: Design of antenna array front end at $24 \mathrm{ghz}$. In Antennas and Propagation (EUCAP), Proceedings of the 5th European Conference on, april 2011.

[20] K. Topalli, O.A. Civi, S. Demir, S. Koc, and T. Akin. A monolithic phased array using 3-bit distributed rf mems phase shifters. Microwave Theory and Techniques, IEEE Transactions on, 56(2):270-277, Feb 2008.

[21] R. Baggen, S. Vaccaro, and D.L. del Rio. Design considerations for compact mobile ku-band satellite terminals. In Antennas and Propagation, 2007. EuCAP 2007. The Second European Conference on, pages $1-5$, nov. 2007

[22] A. Stark, A. Dreher, H. Fischer, A. Geise, R. Gieron, M. Heckler, S. Holzwarth, C. Hunscher, A.F. Jacob, K. Kuhlmann, O. Litschke, D. Lohmann, W. Simon, F. Wotzel, and D. Zahn. Santana: Advanced electronically steerable antennas at ka-band. In Antennas and Propagation, 2009. EuCAP 2009. 3rd European Conference on, pages $471-478$, march 2009.

[23] S. Holzwarth, A. F. Jacob, A. Dreher, C. Hunscher, H. Fischer A. Stark, B. Rohrdantz, A. Geise, K. Kuhlmann, R. Gieron, O. Litschke, D. Lohmann, W. Simon, P. Buchner, M. V. T. Heckler, and L. A. Greda. Active antenna arrays at ka-band: Status and outlook of the santana 
project. In Antennas and Propagation (EuCAP), 2010 Proceedings of the Fourth European Conference on, pages 1 -5, april 2010.

[24] M. Geissler, F. Woetzel, M. Bottcher, S. Korthoff, A. Lauer, M. Eube, and M. Wleklinski. L-band phased array for maritime satcom. In Phased Array Systems and Technology (ARRAY), 2010 IEEE International Symposium on, pages $518-523$, oct. 2010 .

[25] J.G. Trujillo, M.S. Natera, I. Montesinos, M.A. Campo, M.S. Perez, and R. Martinez. Geoda-grua: Adaptive multibeam conformal antenna for satellites communications. In General Assembly and Scientific Symposium, 2011 XXXth URSI, pages 1 -4, aug. 2011.

[26] J. Butler and R. Lowe. Beam-forming matrix simplifies design of electronically scanned antennas. Electronic Design, 9:170-173, 1961.

[27] J. Blass. Multi-directional antenna - new approach top stacked beams. IRE International Convention record, pages 48-50, 1960.

[28] G. Expósito-Domínguez, P. Padilla-Torre, J. M. Fernández-González, and M. Sierra-Castañer. Dual circular polarized steering antenna for satellite communications in $\mathrm{x}$ band. Progress In Electromagnetic Research, 122:61-76, 2012.

[29] Morin G. A. Tang M. Q. Richard S. Rao, K.S. and K. K. Chan Development of a $45 \mathrm{ghz}$ multiple-beam antenna for military satellite communications. IEEE Transactions on Antennas and Propagation, 43(10), Oct 1995.

[30] C.H. Walter. Traveling Wave Antennas. McGraw-Hill, 1965.

[31] J.N. Hines, V.H. Rumsey, and C.H. Walter. Traveling-wave slot antennas. Proceedings of the IRE, 41(11):1624-1631, Nov 1953. 